\title{
Comunidades epistémicas en la solución de problemas ambientales. Tendencias en la recuperación de ríos urbanos
}

\section{Epistemic communities on environmental problem-solving. Trends in urban rivers restoration}

doi: http://dx.doi.org/10.32870/

espiral.v25i71.6106

\begin{abstract}
Resumen
El objetivo del presente artículo es subrayar la dimensión social y política de las iniciativas en torno a la restauración de ríos urbanos. Se afirma que en torno a la contaminación de un río hay una pluralidad de marcos cognitivos que la interpretan desde diferentes perspectivas. Para abordar dicha pluralidad, se recurre al concepto de comunidad epistémica. Se concibe que la consolidación de una comunidad epistémica radica en la apertura y continuidad de espacios deliberativos. La propuesta teórica se ilustra con los diferentes marcos cognitivos, surgidos principalmente de entrevistas a actores, que se identifican en la iniciativa gubernamental de recuperación del río Magdalena, en la Ciudad de México.
\end{abstract}

Palabras clave: comunidad epistémica, río urbano, participación, marco cognitivo, deliberación.
Itzkuauhtli Zamora Saenz
Keywords: Epistemic community, urban river, participation, cognitive frame, deliberation.

-Becario posdoctoral del Instituto de Investigaciones Sociales (IIS), Universidad Autónoma de México (UNAM), México. ORCID: http://orcid.org/0000-0002-1554-6984.

itzaben@gmail.com

Fecha de recepción: 08 de septiembre de 2016. Fecha de aceptación: I4 de agosto de 2017. 


\section{Introducción}

El río La Coyotera, ubicado en el municipio mexiquense de Huixquilucan, se desbordó el 18 de julio de 2016 por las lluvias torrenciales que se precipitaron en la zona. El cauce había sido previamente entubado para construir encima del lecho un área verde de uso privado para los condominios aledaños. La acumulación de basura aguas arriba y un ducto muy reducido provocaron el taponamiento del tubo y su posterior desbordamiento, lo cual provocó la caída de muros, así como la inundación de estacionamientos y casas habitación aledañas. Este hecho fue interpretado de dos maneras distintas por los habitantes del lugar: para unos, evidenció la urgencia de entubar el tramo que todavía está a cielo abierto como la única obra posible de mitigación que previniera el desbordamiento; para otro sector, la inundación mostró la memoria de un río que recuerda su cauce natural, de manera que la mejor política de manejo consistiría en desentubarlo completamente y recuperar su funcionamiento hidrológico (Cruz, 2016).

Estas dos visiones tan opuestas en torno al manejo de los ríos urbanos son una constante en la mayoría de las ciudades de nuestro país: mientras unas voces insisten en entubarlos para evitar inundaciones y focos de infección por el vertimiento de aguas residuales y la acumulación de basura (paradigma sanitarista), otras promueven la importancia de recuperarlos para convertirlos en espacios públicos con servicios ambientales y urbanos para toda la metrópoli (paradigma socioecológico). Aunque internacionalmente el paradigma socioecológico en el manejo de ríos ha ido ganando cada vez mayores adeptos como parte de la gestión integral de los recursos hídricos, las iniciativas relativas a este paradigma resultan muy complejas de implementar porque requieren una importante coordinación entre autoridades políticas y comunidades locales 
(Bernhardt y Palmer, 2007; Everard y Moggridge, 2012; Thoms, et al., 2016).

En ese sentido, la restauración de ríos urbanos puede considerarse como un problema perverso (Rittel y Webber, 1973), ya que su diseño e implementación implican tener en cuenta una gran cantidad de variables involucradas ${ }^{1}$ y una toma de decisiones con información incompleta y, además, con intereses y percepciones sociales contradictorios sobre lo que se debe hacer. Estas características intrínsecas de los problemas perversos provocan que la decisión final siempre deje personas insatisfechas. Se puede decir que la mayoría de los problemas ambientales son perversos, ya que alrededor de cada uno coexiste una pluralidad de percepciones y valoraciones para definirlo y para establecer una alternativa de solución, es decir, en torno a un problema ambiental es posible identificar diferentes marcos de significado con los que se interpreta. ${ }^{2}$

De hecho, un problema ambiental lo es sólo cuando un grupo lo reconoce como tal y busca extender socialmente su marco para que otros sectores compartan la urgencia de solucionarlo. Es verdad que el debate sobre la posible existencia de una realidad biofísica independiente de la dimensión simbólica y cognitiva sigue estando muy presente en la definición de los problemas ambientales, pero incluso posturas como el postpositivismo o el realismo crítico han

I. Mejoramiento de la calidad del agua, restauración de vegetación riparia, recuperación de la planicie de inundación, apertura de espacios públicos asociados, entre otras.

2. En este artículo se utiliza el concepto de marco de significado desde la definición propuesta por Erving Goffman, esto es, como la manera en que un sujeto define una situación determinada a partir de la interacción social predominante en su vida cotidiana y con la cual organiza su experiencia. El marco le permite al sujeto enfrentar problemas que emergen en su entorno y que le plantean la necesidad de responder a la pregunta “iqué está pasando aquí?”. En otras palabras, el marco es una interpretación de la realidad que consiste en la selección y codificación de situaciones o eventos que le permiten al sujeto clasificar y actuar en el mundo (Goffman, 1975). 
aceptado el papel activo que tienen las construcciones sociales en la percepción y valoración del entorno (Burningham y Cooper, 1999; Goldman y Schurman, 2000; Kuhn, 1991; Woodgate y Redclift, 1998).

En el presente artículo, se desarrolla el concepto de comunidad epistémica para analizar el diálogo que sostienen diferentes actores sociales con el objetivo de definir un problema determinado y alcanzar una solución compartida. En su construcción, se van entablando diferentes relaciones sociales que permiten el intercambio de información y la generación de consensos entre los marcos cognitivos. Para que esto sea posible, es indispensable que la comunidad epistémica interactúe a lo largo del tiempo para articularse como una red con cierta estabilidad que favorezca la acción colectiva.

En la primera sección del presente trabajo, se desarrolla la propuesta conceptual de la comunidad epistémica y la importancia que representan los espacios deliberativos para su construcción.

En la segunda parte, se aborda la manera en que las comunidades epistémicas se pueden constituir en torno a la recuperación de ríos urbanos, y se contrasta el entramado teórico a partir de un caso fallido: la recuperación del río Magdalena, en el surponiente de la Ciudad de México; se identifican los principales marcos cognitivos con los cuales se interpretaron la contaminación y la posible recuperación del río, así como las decisiones que impidieron consolidar una comunidad epistémica comprometida con esta causa.

A pesar de que el caso no es el mejor para ilustrar la emergencia de una comunidad epistémica en torno a la recuperación de un río urbano, los marcos cognitivos que se identificaron pueden ser de utilidad para comprender procesos similares en otras cuencas urbanas del país en donde la población se debate entre el entubamiento o la restauración. 


\section{I.El surgimiento de comunidades epistémicas}

En las últimas dos décadas, es posible atestiguar una proliferación de modelos para la toma de decisiones políticas que abogan por la necesidad de crear mecanismos incluyentes que promuevan la participación multisectorial. Estas propuestas han recibido los nombres de ciencia posnormal, planeación participativa o gobernanza, por mencionar algunos de distintas corrientes (Funtowicz y Ravetz, 1993; Huitema, et al., 2009; Schmoldt, Kangas y Mendoza, 2001).

La premisa general de estas propuestas es que la solución de problemas complejos como los ambientales no puede ser resultado de una decisión exclusivamente técnica o científica que se imponga de manera jerárquica y vertical: requiere incluir el conocimiento y la postura de las comunidades locales que serán las principales beneficiadas o perjudicadas por la implementación de la política. Bajo esta perspectiva, tanto las decisiones como los procesos para tomarlas son igualmente importantes, esto es, no importa solamente alcanzar un buen resultado, sino que su formulación se haya dado bajo mecanismos abiertos, plurales e incluyentes.

Estos modelos participativos coinciden con lo que Mark Warren ha denominado democracia expansiva, la cual se caracteriza por la ampliación de mecanismos participativos en pequeña escala y por la necesidad de incluir en las soluciones la voz de los posibles afectados por las decisiones gubernamentales (Warren, 1992). Esto implica que una gestión democrática del territorio no se reduce a la legitimidad que la clase política obtiene por la elección popular, sino que también descansa en los procesos deliberativos abiertos a escuchar la voz de todos los interesados. Las instituciones clásicas de la democracia representativa siguen teniendo sentido e importancia, pero resultan insuficientes para coordinar la acción de diferentes actores que buscan resolver problemas perversos. 
Hay una gran cantidad de mecanismos específicos para favorecer la participación multisectorial como talleres de planeación, foros, mesas de participación, grupos promotores, paneles de expertos o consejos ciudadanos, por mencionar algunos. Todos estos mecanismos comparten la idea regulativa de favorecer el entendimiento común y la cooperación entre diferentes actores. Peter Haas (1992) acuñó el concepto de comunidad epistémica para referirse a un grupo de personas que se reúnen periódicamente para generar acuerdos sobre un problema y su solución. El concepto lo utilizó Haas por primera vez para referirse a la emergencia de una red de actores gubernamentales, civiles y académicos de diferentes países que se congregaron para intercambiar información con el objetivo de revertir la contaminación del mar Mediterráneo.

El propio Haas agregó que el tipo de problemas que pretende resolver una comunidad epistémica se caracteriza por estar inserto en un contexto de incertidumbre en el que los diferentes actores reconocen que el conocimiento científico no elimina el riesgo de consecuencias no deseadas de una decisión determinada, sino que incluso los puede hacer más conscientes de las posibles secuelas (Haas, 2004).

Las comunidades epistémicas no están circunscritas a una demarcación territorial de carácter administrativo (estado, municipio o ciudad), sino que varían de acuerdo a la escala del problema que se pretende solucionar, por lo que su conformación tiene el desafío de incluir a una pluralidad de actores políticos con atribuciones administrativas y orientaciones ideológicas distintas.

El ejemplo por excelencia para ilustrar esta idea son las políticas para combatir el cambio climático. Los avances en la consolidación de esta comunidad epistémica tienen como objetivo central alcanzar una solución en una escala global, ya que no tendría sentido que un país o un municipio actúe de manera aislada a un problema de esta magnitud. En 
dicha comunidad, convergerán una gran cantidad de marcos cognitivos sobre el cambio climático (incluso Gobiernos que nieguen el problema), pero, teóricamente, si hay una continua interacción entre los diferentes actores, aumentará la propensión a tomar decisiones colectivas resultadas del consenso y la cooperación (Giddens, 2010; Gough y Shackley, 2001). Un ejemplo de esta posibilidad es el Acuerdo de París, que se alcanzó en el año 2015 y que fue el resultado de negociaciones entre representantes de ciento noventa y cinco países para definir acciones conjuntas que permitirían disminuir las emisiones de gases de efecto invernadero que contribuyen al calentamiento global. Incluso, la reciente decisión de Donald Trump de retirar a Estados Unidos de este acuerdo es evaluada en el marco de las metas y objetivos establecidos por dicha comunidad epistémica (ONU, 2015).

El diálogo que entabla una comunidad epistémica está dirigido prioritariamente a generar consensos para definir y compartir los marcos cognitivos con los que sus integrantes perciben los problemas en cuestión, así como las alternativas para solucionarlos. En este punto es importante hacer una aclaración sobre los marcos de significado. Estos no se constriñen a un aspecto meramente interpretativo sobre la realidad, sino que tienen un sustrato pragmático que orienta las prácticas y permite formular acciones o planes de acción para modificarla (Sawyer, 2005). La representación que hace un sujeto está orientada a la acción, por lo que constituye el basamento de prácticas y usos sociales sobre el territorio. Es cierto que los procesos de comunicación y negociación que entablan los participantes no están destinados a lograr una completa unidad de los diferentes marcos, pero contribuyen sensiblemente a que disminuyan las distancias cognitivas gracias al reconocimiento de otras perspectivas sobre el tema, a la escucha de diferentes argumentos y al desarrollo de un sentido de corresponsabilidad en la recuperación ambiental (Fung y Wright, 2001; Hajer y Wagenaar, 2003; Wagenaar, 
2006). De lograrse estos acuerdos, emerge una identidad colectiva -nosotros- que busca superar un problema común a partir de estrategias compartidas.

La información que circula en la comunidad epistémica lo hace en diferentes direcciones. En general, hay una mayor predisposición a escuchar y aceptar los datos que provienen del ámbito académico, pero la ciudadanía también suele aportar conocimiento local sobre el problema, mientras que los funcionarios a su vez pueden sumar una gran cantidad de información en la medida en que hagan pública la literatura gris que generan las instituciones gubernamentales y que en muchas ocasiones se queda almacenada en los archivos sin que constituya un elemento central para la gestión territorial. Este último flujo suele ser uno de los más complicados de realizar, ya que en ocasiones la información es utilizada por los funcionarios como un mecanismo de poder que les permite preservar su puesto a lo largo de diferentes administraciones (Allison y Zelikow, 1999). La resistencia del funcionario también puede ser resultado de una acción deliberada para ocultar un posible conflicto de interés.

No obstante, la reserva a compartir información no es exclusiva de este actor: también es creciente esta actitud en la ciudadanía, pues esta considera que su contribución tiene una nula efectividad en la toma de decisiones que instrumenta el Gobierno. ${ }^{3}$ También, y a pesar de que la ciencia tiene como una premisa básica hacer públicos los métodos y

3. Es importante advertir que las comunidades locales también han aumentado su recelo a compartir información con académicos. Aunque generalmente los científicos suelen ser considerados como aliados estratégicos en luchas sociales por la posibilidad de que generen información que legitime su demanda o que incluso visibilice socialmente el problema que viven en su territorio, también perciben que esta responsabilidad social no es compartida por todos los científicos, ya que algunos no comparten los resultados de su investigación con la comunidad en la que trabajaron. Estos investigadores que, podría pensarse,"toman notas, miden y se van", dejan un precedente negativo con las comunidades locales y constituyen una llamada de atención para reflexionar sobre la importancia que implica seguir el principio de reciprocidad -devolver algo a la comunidad-como parte de la práctica investigativa. 
los resultados de la investigación, algunos académicos son recelosos a compartir información que no han publicado.

Además de las diferentes resistencias que los actores presentan para compartir la información, hay otro proceso intrínseco aún más complicado en la creación de una comunidad epistémica: compartir el sentido del dato. El dato puede definirse como una información cualitativa o cuantitativa sobre la realidad que depende de las teorías, el conocimiento práctico y las valoraciones sociales predominantes en un contexto histórico determinado (Bunge, 1999; King, Keohane y Verba, 1994), es decir, cualquier observación sobre la realidad no constituye un proceso únicamente fisiológico, sino que es una construcción en la que intervienen teorías (científicas y no científicas), conjeturas y valores (Kuhn, 1991; Norwood, 1969). En una misma coordenada espacio-temporal, se encuentran diferentes maneras de observar y organizar la realidad. Esta construcción no se hace desde la individualidad, sino desde y para una colectividad creadora de sentido (Searle, 1997).

La información que integra los marcos cognitivos no se limita a la dimensión tangible o biofísica del territorio, es decir, a su valoración como una extensión física que se puede medir y cuantificar. En los marcos cognitivos también se encuentra una dimensión simbólica, con la cual un grupo interpreta el espacio de acuerdo a su horizonte cultural. Esta dimensión simbólica agrupa los aspectos ideológicos que expresan un sentir comunitario y convierten al territorio en un objeto de afecto y de una valoración especial, como puede resultar de considerarlo el lugar de los antepasados -el espacio de una historia común-, o como recinto sagrado o patrimonio ambiental, por mencionar unos ejemplos.

Dicho con otras palabras: la dimensión simbólica concentra los significados compartidos que le dan un sentido eminentemente social al espacio mediante la creación, circulación y actualización de una forma de vida compartida. Su 
presencia permite trazar sueños, esperanzas y narrativas que posibilitan al ser humano tener una relación particular con los demás y con su entorno (Augé, 2001; Giménez, 2001).

La pluralidad y la heterogeneidad de las sociedades contemporáneas han acentuado la coexistencia de diversos marcos cognitivos en un mismo territorio. Esta característica nos alerta a dejar de considerar una cuenca (o cualquier unidad geográfica) como un espacio homogéneo que se expresa de la misma manera en cada uno de los individuos o instituciones que ahí se encuentran. Bajo esta falsa creencia, bastaría con entrevistar a una persona o estudiar a una sola institución para conocer la dimensión simbólica de un territorio. Por el contrario, resulta fundamental identificar los diferentes marcos cognitivos, las jerarquías sociales y la complejidad de su organización para vislumbrar su funcionamiento, reproducción, o bien, su propia transformación.

Ejemplifiquemos esta idea con el caso de los ríos. El criterio para delimitar espacialmente un río consiste en el parteaguas, es decir, una línea imaginaria que une los puntos de mayor elevación de un terreno para identificar a todos los escurrimientos que desembocan y forman una corriente de agua superficial, misma línea que sirve para separar y establecer los límites con respecto a las cuencas contiguas. Los vecinos del río generalmente se relacionan y perciben el río desde una aproximación diferente que no considera la escala de la cuenca: es una fuente de agua, un espacio para la recreación y la convivencia social, un elemento indeseable del paisaje por su fuerte contaminación o incluso un geosímbolo que forma parte de una identidad colectiva local o regional (Bonnemaison, 1981).

$\mathrm{Al}$ resaltar los diferentes significados que hay sobre un río, su percepción social va más allá de la definición científica acerca de una realidad objetiva para incluir diferentes relaciones y miradas sociales. Recuperando una frase de Gregory Bateson, se puede afirmar que sobre un río (y en 
realidad sobre cualquier ecosistema) hay una ecología de significados que se refieren a lo que es, a lo que representa y a lo que se puede hacer con él (Bateson, 2011). Cuando se recogen los diferentes marcos de significado sobre un río, resalta cómo algunos son próximos entre sí, mientras que otros resultan tan alejados que es difícil aceptar que se refieren al mismo río. Por esta razón, la creación de una comunidad epistémica no es un proceso automático, sino que requiere de un diálogo continuo y de una real apertura a escuchar otras voces por parte de cada uno de los actores. ${ }^{4}$

Cuando se afirma que las comunidades epistémicas están conformadas por grupos heterogéneos que representan marcos cognitivos distintos, se infiere que sus procesos internos, a pesar de estar orientados a la cooperación, son fundamentalmente conflictivos. La relación de los marcos en la construcción de una comunidad epistémica no está mediada por un diálogo neutro y simple: expresa las tensiones derivadas entre los intereses y valores de los grupos sociales. Cada uno pretende que su visión sea la hegemónica, de manera que regule y establezca las prácticas socialmente aceptadas para percibir y habitar el territorio. La construcción de puentes entre los diferentes marcos de significado favorece las posibilidades para generar una acción colectiva lo más consensuada posible que refleje

4. Incluso en el ámbito académico, la emergencia de una comunidad epistémica en torno a la resolución de un problema público es un proceso difícil de lograr debido a los diferentes paradigmas y corrientes teóricas que hay al interior de una misma disciplina. Esta característica, mucho más notoria en las ciencias sociales, establece fronteras epistémicas desde las cuales se construye y se valora el objeto de estudio (Cortés, 2008). Si la inmensurabilidad de las tradiciones de investigación al interior de una misma disciplina resulta difícil de superar, esta disonancia cognitiva aumenta cuando el diálogo es multidisciplinario (García, 2000).Aunque todos los participantes puedan compartir un ideal regulativo de inicio (digamos, restaurar un río), lograr un consenso de cómo hacerlo implica un trabajo colaborativo sostenido para comprender la manera en que una disciplina distinta a la propia delimita y aborda el problema en cuestión, así como para acordar estrategias compartidas por todos los participantes (Perló y González, 2009). 
las metas y objetivos políticos que trazó para sí misma la comunidad epistémica (Schön y Rein, 1994).

En este sentido, se puede afirmar que las comunidades epistémicas presentan una dinámica dialógica entre la cooperación y el conflicto. Las comunidades son cooperativas porque las relaciones entre sus miembros se entablan bajo la premisa de que la solución de los problemas a los que se enfrentan requiere de la participación y la colaboración multisectorial, meta para la cual se transita por un periodo caracterizado por el conflicto, desde la propia definición del problema hasta la propuesta de diversas alternativas de solución. Otro elemento a considerar es que los integrantes de una comunidad epistémica tendrán una distribución desigual de recursos (por ejemplo, influencia y dinero) para hacer valer sus intereses (Hajer y Wagenaar, 2003).

Ahora bien, el conflicto intrínseco de las comunidades epistémicas tiene un gran poder analítico si se aborda desde su productividad social (Azuela y Cosacov, 2013; Melé, 2006). En esta vertiente, el conflicto consiste en un vínculo social con efectos visibles en una colectividad a partir de que dos o más actores persiguen intereses contrarios con respecto a un proceso o un recurso determinado. El conflicto constituye una oportunidad para que los involucrados actualicen los acuerdos que regulaban las acciones en un campo social. ${ }^{5}$ Esto no significa que los nuevos acuerdos alcanzados sean mejores que los anteriores, pero es indudable que esa esfera es trastocada y abre un nuevo proceso de análisis capaz de alterar algunas reglas de la colectividad.

Por lo anterior, se infiere que la comunidad epistémica no se integra por el mero hecho de reunir esporádicamente a diferentes actores sociales interesados en resolver un problema, sino que se alcanza en la medida en que puede

5. Al respecto, Coser afirma que "Al suscitar nuevas situaciones, que no están definidas parcial o totalmente por reglas y normas, el conflicto actúa como un estímulo para establecerlas" (Coser, 196I, p. I4I). 
mantenerse la interacción entre los diferentes participantes. En ese sentido, los espacios deliberativos juegan un papel fundamental en la construcción de comunidades epistémicas.

El principal objetivo de los espacios deliberativos en la construcción de comunidades epistémicas, además de favorecer la voz de diferentes actores interesados en una decisión sobre un asunto público, consiste en ser el mecanismo que permite alcanzar guías cognitivas para el logro de acuerdos. La participación en estos espacios les permite a los actores completar la información que tienen sobre el tema a partir de las relaciones sociales que entablan entre ellos. En estas conversaciones, los actores redefinen o corroboran su posición sobre el tema a partir de la información que circula al interior de la comunidad.

Los espacios deliberativos que abre el Gobierno o que exige la ciudadanía constituyen un mecanismo que favorece la disminución de los intereses particulares en aras de lo que procesualmente se va identificando como un bien público, es decir, el bien público no es algo predeterminado o que define exclusivamente un grupo social, aunque discursivamente así lo declare cada uno de los grupos participantes: en realidad, es el resultado del proceso deliberativo, en el cual se delinean ciertos acuerdos sobre el problema que convoca a los actores y sobre las mejores alternativas para solucionarlo. Con esta premisa, los espacios deliberativos se convierten en el mecanismo por excelencia para lograr la acción colectiva de una comunidad epistémica, sobre todo si logran funcionar con regularidad.

La apertura de los espacios deliberativos tiene como consecuencia que los diferentes miembros de la comunidad epistémica reconozcan la contingencia de sus decisiones, es decir, que identifiquen las diferentes opciones para orientar la intervención territorial. En otras palabras, como resultado de la deliberación, se reconoce una variedad de 
opciones que se descartaron para elegir una. Por esta misma razón, aunque los espacios estén orientados a la búsqueda de consensos, los disensos -otras opciones que se pusieron sobre la mesa y que fueron eliminadas- no desaparecen en su totalidad, sino que esperarán oportunidades para expresarse nuevamente. El consenso es una meta de los espacios deliberativos, pero no es una obligación o una consecuencia inevitable de su apertura.

\section{Ecología de significados en la recuperación de ríos urbanos}

La mayoría de los ríos del mundo tiene un grado importante de alteración ecológica, ya sea por la contaminación del cauce debido al vertimiento de aguas industriales o residuales, por la ocupación de la planicie de inundación resultada del crecimiento urbano, o bien, por la segmentación del cauce debido a la construcción de grandes presas para la generación de energía hidroeléctrica o para la irrigación (Gurnell, Lee y Souch, 2007; McCully, 2001; Postel y Richter, 2003; Thoms, et al., 2016).

Es posible afirmar que el manejo de los ríos durante el siglo xx estuvo directamente relacionado con su tamaño: los grandes ríos fueron considerados ejes para el desarrollo y la modernización de los países mediante la construcción de infraestructura hidráulica que lograba captar mayor cantidad de agua para las actividades humanas (White, 1995; Worster, 1992), mientras los pequeños ríos fueron percibidos como un elemento indeseable del paisaje urbano y utilizados como drenajes de las viviendas que se asentaron en las riberas. La solución modernizadora consistió en entubarlos y expulsar su agua mediante el sistema de drenaje lo más lejos de la ciudad para evitar problemas de salud pública (Tucci, 2012). En ambos casos, se alteró considerablemente el régimen hídrico de los ríos. 
En las últimas dos décadas, han proliferado iniciativas que promueven la restauración integral de los ríos, es decir, que no se limitan a una intervención cosmética del cauce mediante el mejoramiento de la calidad del agua, sino que pretenden recuperar sus funciones ecosistémicas y su régimen hídrico natural (Bernhardt y Palmer, 2007; Clifford, 2007; Riley, 1998). Hay una corriente de estas iniciativas que se ha especializado en los ríos urbanos, esto es, en los ríos cuya cuenca presenta un grado considerable de superficie impermeable, resultado de la infraestructura de las ciudades (Findlay y Taylor, 2006; Petts, Heathcote y Martin, 2002).

Estos proyectos de política pública son verdaderos problemas perversos, en el sentido anteriormente mencionado, ya que en una cuenca urbana coexisten diferentes actores con usos, prácticas y marcos cognitivos distintos sobre el río, además de que el conocimiento que se tiene sobre el sistema socioecológico es incompleto por la gran cantidad de procesos involucrados en su funcionamiento.

Si se realiza una tipología general sobre los actores presentes en una cuenca urbana, es posible identificar a los siguientes: 1) las comunidades rurales que habitan el suelo de conservación y que pueden utilizar el agua del río con fines de irrigación; 2) los actores urbanos que radican en la zona consolidada de la ciudad y que pueden proveerse de agua mediante su potabilización, además de incorporar el cauce al paisaje urbano como un espacio público para la recreación y la convivencia social; 3) los representantes del Gobierno encargados de gestionar los recursos hídricos del río, su infraestructura hidráulica asociada, así como otros elementos socioecológicos de la cuenca como el suelo de conservación, la vegetación riparia y el patrimonio arquitectónico vinculado al río.

Es importante subrayar que esta tipología es muy general y no pretende agotar la heterogeneidad de actores que 
pueden involucrarse en la construcción de una comunidad epistémica. De acuerdo al caso específico que se aborde, será muy importante identificar otros actores cuyos marcos pueden ser fundamentales para recuperar los significados más importantes que inciden tanto en el estado actual del río como en el planteamiento de alternativas de solución.

Por ejemplo, en ocasiones han sido las iniciativas de artistas las que han logrado visibilizar la contaminación de un río y promover la urgencia de una iniciativa para recuperarlo (Giannachi y Stewart, 2005; Heim, 2003; Wallen, 2012; Zamora, 2016). En otras, la variable étnica puede resultar fundamental para entender la disonancia cognitiva entre diferentes grupos sociales, ya que los lenguajes de valoración llegan a ser más distantes entre sí, al grado de encontrar significados inconmensurables que dificultan el consenso. En esta vertiente destacan las luchas de grupos indígenas que se oponen a la construcción de grandes presas en su territorio o que denuncian la contaminación provocada por la industria en los ecosistemas fluviales (Martínez Alier, 2009).

En cualquier caso, las experiencias en las que se ha logrado revertir la contaminación de un río coinciden en haber incluido a los actores relevantes que influyen en el estado ambiental de la cuenca, coordinando y regulando sus acciones. Es cierto que la recuperación de un río urbano no se restringe a un problema de coordinación multisectorial, pero sin ella es muy complicado lograrla.

Ahora bien, no existe un solo modelo de coordinación multisectorial para estos proyectos: cada iniciativa requiere tener en cuenta la historia y la tradición política local para impulsar arreglos institucionales que puedan funcionar (Warner, Wester y Bolding, 2008). No obstante, dentro de estos arreglos parecen fundamentales aquellos relacionados con la explicación de las reglas de representación y las de participación en la toma de decisiones (Jaspers, 2003). 
A continuación, se expone la manera en que se intentó construir una comunidad epistémica en el marco del plan maestro para la recuperación del río Magdalena, ubicado en la Ciudad de México. Aunque se puede considerar como una historia fallida en la consolidación de una comunidad epistémica en torno al río, la iniciativa resulta de interés porque brinda la posibilidad de identificar los marcos de significado más importantes que se expresaron en los espacios deliberativos. También es útil para reflexionar en torno a los obstáculos que existieron para conformarla, más allá de la intrínseca dificultad de alcanzar consensos entre los diferentes participantes.

\section{La recuperación del río Magdalena en la Ciudad de México}

La Ciudad de México ha sido ejemplar en el manejo sanitarista de ríos urbanos. Con la desecación del sistema lacustre en que fue fundada, los ríos pronto constituyeron un elemento ilógico de la cuenca, ya que no desembocaban en ningún lugar. El crecimiento exponencial de la ciudad durante el siglo xx no contempló seriamente la alternativa de preservar los lagos y el sistema fluvial asociado, aunque no se puede olvidar que sí han existido otros proyectos que han promovido un manejo hídrico más sustentable (González de León, et al., 2010). Sin embargo, se consideró que el mejor uso que podían tener los ríos era el drenaje, o entubarlos y transformarlos en vialidades (González, Hernández, et $a l ., 2010)$. Tal vez la mayoría de la población capitalina no tenga presente que las actuales avenidas Viaducto-Piedad, Churubusco, Mixcoac, San Joaquín y Consulado fueron ríos hace menos de un siglo. A pesar del predominio de este modelo sanitarista de los ríos, hubo algunos de ellos que corrieron con mejor suerte: el río Magdalena, el río de 
los Remedios, el río Ameca y el Santo Desierto no fueron entubados en su totalidad (Legorreta, 2009).

Hoy en día, el río Magdalena es el único río que participa en el abastecimiento de agua potable de la Ciudad de México. Ubicado al surponiente de la urbe, el río recorre $14.8 \mathrm{~km}$ por el suelo de conservación hasta llegar a una planta potabilizadora que aprovecha 200 litros por segundo del caudal para distribuirlos en diferentes colonias de la delegación Magdalena Contreras. Después de la planta, el régimen fluvial es un auténtico híbrido, ya que mientras en estiaje sólo transporta agua residual, en temporada de lluvias incrementa considerablemente su gasto y llega a desbordarse.

A lo largo de los $13.4 \mathrm{~km}$ del río que se internan en la ciudad, su cauce se encuentra canalizado y parcialmente entubado, y su tramo final corre a cielo abierto. En este último sector, el río cruza por los viveros de Coyoacán, un área verde altamente frecuentada por los pobladores de la ciudad, y luego confluye con el río Mixcoac para formar el río Churubusco, el cual se encuentra totalmente entubado y convertido en una importante vialidad.

En el año 2007, la Secretaría del Medio Ambiente (Sedema) del entonces Gobierno del Distrito Federal (GDF) comenzó una iniciativa para la recuperación integral del río. Fue la primera iniciativa que establecía como su objetivo la intervención a lo largo de todo el cauce para convertirlo en un espacio público importante para la ciudad y para la recuperación de sus servicios ecosistémicos.

Desde el comienzo, se planteó la importancia de incluir en la iniciativa la participación de vecinos, comunidades rurales, instancias académicas, organizaciones de la sociedad civil y dependencias de Gobierno, tanto delegacionales como del GDF. Durante los años 2007, 2008 y 2009, se implementaron diferentes mecanismos para incentivar la participación ciudadana, entre ellos la conformación del Grupo 
Promotor para el Rescate de la Cuenca del río Magdalena y la realización de talleres de planeación participativa para retroalimentar el Plan maestro para el manejo integral y el aprovechamiento sustentable del río Magdalena (Sedema y UNAM, 2009).

Mientras que el grupo promotor tuvo una convocatoria abierta a cualquier interesado en el río, los talleres fueron más focalizados: tres de ellos se dirigieron a canalizar la participación del núcleo agrario de la Magdalena Atlitic, cuyos bienes comunales coinciden prácticamente con el suelo de conservación de la cuenca, mientras que otros dos se organizaron para recuperar la voz de vecinos de colonias de la zona del Pedregal y las aledañas a los viveros de Coyoacán. Estos últimos talleres contaron con una escasa asistencia.

A partir del análisis documental que se hizo de la memoria estenográfica de esos talleres, y de entrevistas a profundidad con actores que participaron en estos mecanismos, se reconstruyeron para esta investigación los marcos cognitivos que se expresaron en torno a la recuperación del río Magdalena. ${ }^{6}$

A continuación, se desarrolla el marco cognitivo de los actores de Gobierno, en particular de los funcionarios de

6. En total se realizaron veintidós entrevistas semiestructuradas a personas que participaron en los mecanismos de planeación participativa del plan maestro y con algunas autoridades de Gobierno que formaron parte del proceso. Las entrevistas para construir los marcos cognitivos se distribuyeron de la siguiente manera: seis entrevistas a funcionarios de Gobierno, ocho a comuneros de La Magdalena Atlitic y ocho con residentes de la colonia Chimalistac y de La Magdalena. Es importante subrayar que esta información se complementó con el análisis documental de la memoria estenográfica de los talleres de planeación participativa que se realizaron en el marco de la elaboración del plan maestro del río Magdalena. Las entrevistas fueron transcritas y posteriormente analizadas con ayuda del programa ATLAS.ti (versión 6.0.15) con el objetivo de identificar las categorías más relevantes de cada marco (actor de Gobierno, actor rural y actor urbano). La estrategia para construir los marcos cognitivos siguió el procedimiento expuesto en la teoría fundamentada (Strauss y Corbin, 2002) para encontrar los códigos principales presentes en el discurso utilizado por cada tipo actor. Posteriormente, se agruparon, compararon y se refinaron los códigos para obtener las categorías centrales con las que los actores definían el problema del río y su vínculo social con el mismo. 
la Sedema, quienes eran los responsables de coordinar la participación ciudadana alrededor de esta política pública. Posteriormente, se presentan los marcos de los actores que participaron en los talleres de planeación participativa. Estos se dividen en dos grupos: aquellos en los que participaron los comuneros de La Magdalena Atlitic (talleres uno, dos y cinco) y aquellos en los que fueron convocados actores urbanos (talleres tres y cuatro). El análisis de los marcos se realiza desde una perspectiva dinámica que permite destacar los cambios que existieron (en caso de haber sucedido) a lo largo del tiempo, para evaluar si los espacios deliberativos favorecieron consensos entre los diferentes actores involucrados.

\section{I. El marco cognitivo del Gobierno}

La principal característica revisada en este marco consistió en que este actor considera la cuenca como un espacio en el que puede ejercer su poder de decisión durante su mandato. Este poder lo significó discursivamente de diferentes maneras: para proteger el bien común, para fomentar la gestión democrática, para transformar la manera en que la ciudad se relaciona con sus ríos, para construir espacios públicos, por señalar las más importantes.

No obstante, su atribución fundamental radica en su capacidad para elaborar y ejecutar políticas públicas gracias a la generación de conocimiento que permite a este actor identificar, clasificar y ordenar los elementos físicos del territorio. En ese sentido, justifica la necesidad de extender sus marcos a partir de su poder para intervenir el río mediante la información privilegiada que generan sus instituciones. Además, respalda sus intervenciones territoriales con el aparato jurídico y legal existente, lo que constituye la máxima expresión del ejercicio de su poder en el territorio, ya que en dicho entramado se expresan las reglas de usos prohibidos y permitidos (es lo que marca la 
ley), las sanciones respectivas por su incumplimiento y la identificación de los actores reconocidos para participar en el manejo y aprovechamiento de los recursos hídricos (tenemos que seguir la normatividad para actuar en la cuenca).

En los espacios deliberativos, los representantes del Gobierno significaron al río como un elemento indispensable para el funcionamiento hídrico de la ciudad, pero que se encuentra sumamente contaminado, por lo que sus servicios ecosistémicos están en riesgo de perderse. También lo enmarcaron como un río de fuertes contrastes, con agua limpia en el suelo de conservación y sumamente contaminado al interior de la ciudad. Estos elementos del marco se pueden resumir de la siguiente manera:

- Unidad ambiental importante para el equilibrio ecosistémico de la ciudad. El río Magdalena constituye el principal aporte de un cuerpo de agua superficial local en el abasto de la ciudad. El agua del río es de muy buena calidad y su aprovechamiento es mucho más barato que el de la que se trasvasa mediante el sistema Cutzamala. Este hecho fue de una gran relevancia para este actor, ya que si la recuperación del río tiene éxito, podría ser un modelo a replicarse en otras cuencas locales de la zona metropolitana del Valle de México (ZMVM), para que, junto con otras medidas referentes al manejo integral de recursos hídricos, la ciudad sea más sostenible en su modelo de abastecimiento. Por esta razón, un significado central que buscó subrayar la importancia del Magdalena fue definirlo como el único río vivo de la ciudad. El hecho de que esta afirmación sea falsa (hay más ríos vivos en la cuenca) no es relevante para las intenciones centrales de este actor, el cual pretende resaltar el riesgo de que el Magdalena pierda sus servicios ecosistémicos de manera irreversible.

- Rescatar el río como exhorto político. Es muy amplio el repertorio semántico para referirse a una intervención 
ecológica sobre el río. Incluye vocablos como recuperación, renaturalización, rehabilitación y restauración, por mencionar algunos. La Sedema empleó de manera central la palabra rescate para definir su objetivo de mejorar la calidad del agua y construir espacios públicos vinculados al cauce. En ese sentido, la expresión rescatar el río se utilizó como un exhorto político, casi de carácter moral, que privilegió el significado de salvar un ecosistema natural sobre una definición más técnica que clarificara los alcances de su intervención (restauración o rehabilitación, por ejemplo). Esta selección del concepto no es políticamente neutral, ya que la falta de una definición técnica para comunicar su política pública le permitió al actor Gobierno evitar un compromiso claro sobre los objetivos y las metas del proyecto.

- Un río contrastante: la necesidad de una visión integral. El Gobierno planteó gestionar el río Magdalena desde una visión integral, lo que implicaba dos cosas: realizar intervenciones a lo largo de todo el cauce (desde su nacimiento en el paraje de Cieneguillas hasta su desemboque final en el río Churubusco), y considerar la cuenca como la unidad de planeación, esto es, si bien el rescate del río se enfocaba en el cauce, este actor mencionaba que era indispensable poner atención en los procesos que suceden en el ecosistema asociado. La Sedema se asumió como el único actor que podía caracterizar y gestionar los procesos del río tanto aguas arriba (agua limpia y beneficios ambientales para la ciudad) como aguas abajo, en donde se concentraban los problemas y las externalidades de la contaminación (río convertido en drenaje a cielo abierto).

- El tránsito de un responsable difuso a uno concreto. El Gobierno fue muy cauto durante la etapa de planeación respecto a referirse a un actor en específico como el responsable de la contaminación del río, lo que puede seña-

\section{6}


larse como un esfuerzo por evitar alguna confrontación. En ese sentido, su discurso reificó las problemáticas del río, ya que la acumulación de basura, el vertimiento de aguas servidas en el cauce y la invasión de la planicie de inundación se señalaban como procesos sin actores ni responsables: se enunciaron simplemente como presiones que alteraban el equilibrio ecológico de la cuenca. Esta atribución difusa de responsabilidades se modificó cuando se pasó de la etapa de diseño del plan a la de implementación. En esta segunda fase (años 2011 y 2012), se construyeron colectores marginales y una planta de tratamiento en la parte alta de la cuenca. Frente a la oposición de ciertos vecinos a la realización de estas obras, las autoridades señalaron que los vecinos del río pugnaban por su rescate sin asumirse como parte del problema, y que olvidaban que era el drenaje de sus casas lo que contaminaba el cauce. A su vez, los funcionarios de la administración capitalina que impulsaron el plan de rescate no asumieron ningún tipo de responsabilidad en el deterioro de la cuenca. Desde su perspectiva, la contaminación del río era el resultado de acciones y gestiones pasadas que no habían incorporado seriamente el principio de sostenibilidad como un eje de gobierno o que habían tolerado la invasión a la planicie de inundación del río. Sin embargo, no hubo señalamientos claros sobre las relaciones clientelares vigentes que establecen funcionarios locales de la zona (particularmente de las delegaciones Magdalena Contreras y Tlalpan) y que favorecen el crecimiento de los asentamientos irregulares en el suelo de conservación.

\subsection{El marco de los actores rurales}

Los comuneros de Magdalena Atlitic tienen una presencia histórica en la cuenca, por lo que ellos mismos la significan como el territorio de nuestros antepasados. Este hecho está 
reconocido en el entramado jurídico de la ciudad al considerarlos como pueblo originario. Dicho estatus los convierte en una voz legítima e indispensable en la toma de decisiones sobre el río. Los principales rasgos de su marco cognitivo se sintetizan de la siguiente manera:

- Dueños legítimos del río, pero excluidos de su aprovechamiento. La extensión de los bienes comunales de Magdalena Atlitic coincide casi en su totalidad con el suelo de conservación de la cuenca del río Magdalena. Para los pobladores, el río nace en su territorio y la excelente calidad del agua se mantiene gracias a los trabajos que hacen de conservación del bosque. A pesar de esto, este actor considera que la comunidad tiene una relación paradójica con su entorno, ya que, durante la mayor parte del siglo $\mathrm{xx}$ ha tenido restricciones para aprovecharlo, incluso cuando tiene la propiedad legal del bosque. A partir de 1932, comenzó a desarrollarse un entramado jurídico orientado a la conservación del bosque, iniciando con el decreto presidencial que definió al parque Los Dinamos como zona protectora forestal. Además, con la concesión que realizó en la década de 1970 la Secretaría de Recursos Hidráulicos al entonces Departamento del Distrito Federal para aprovechar el agua del río, los comuneros reafirmaron la percepción de que han sido despojados de la riqueza ambiental de su territorio. Desde su perspectiva, los comuneros no sólo fueron excluidos de la distribución hídrica, sino además tienen una gran cantidad de restricciones para manejar los recursos forestales de la zona, aspecto que relacionan con su situación de pobreza y marginación. Es muy revelador que la iniciativa para recuperar el río no la significaron como una oportunidad para expresar su voz e influir en su manejo, sino que fue valorada principalmente como una nueva iniciativa que impulsaron las autoridades para beneficiarse a sí mismas 
(mediante prácticas de corrupción en la construcción de obra pública), y, en todo caso, para darle más agua a los actores urbanos que no sienten ni conocen el río como los comuneros dicen hacerlo.

- El planteamiento de un destino compartido. La relación histórica que afirman tener los comuneros con el río puede ser interpretada como una especie de simbiosis, de manera que si el río desaparece, ellos correrán con la misma suerte (sin bosque ni agua, la comunidad desaparecerá irreversiblemente), pero también argumentan que si la comunidad desaparece, el río perderá a sus principales defensores y protectores. Este destino compartido legitima en su discurso el derecho a participar en todas las decisiones que involucran el futuro de la cuenca, ya que esto significa defender su propia continuidad como grupo. La radicalización de este planteamiento se reflejó en las descalificaciones que ocasionalmente hacían del sector académico que participó en la elaboración del plan maestro. Para ciertos líderes de la comunidad, sólo a ellos mismos les correspondía el derecho histórico de conocer e investigar los recursos naturales que constituyen su territorio. Este argumento abrió una distancia cognitiva muy importante con el resto de los participantes, ya que varios participantes interpretaron este argumento como un acto de intransigencia que dificultaba la creación de acuerdos. Tiempo después de haber concluido los trabajos del plan maestro, algunos comuneros consideraron que esta actitud de sus líderes dificultó la comprensión de las necesidades y demandas de otros actores interesados en la recuperación del río. No obstante, este elemento sigue siendo de enorme importancia en su marco cognitivo, ya que para los comuneros ellos son los únicos actores que seguirán habitando la cuenca en el futuro: los actores urbanos se pueden mudar y las autoridades de Gobierno se irán 
a otro puesto cuando termine su periodo en la administración. Frente al cambio inminente de autoridades políticas por las elecciones locales que sucederían en el año 2012, los comuneros destacaron que la movilidad de los representantes políticos está directamente asociada a su falta de disposición para hacer inversiones de largo plazo en acciones de carácter ambiental.

- Un río menos caudaloso: relación río-bosque. La presencia histórica de los comuneros en la región les ha permitido atestiguar la contaminación y la disminución del gasto fluvial. Ambos procesos los relacionaron con la falta de mantenimiento del bosque. Por esta razón, en las participaciones que hacían en los talleres, insistieron en que la recuperación tenía que concentrarse en las partes altas de la cuenca, ya que de lo contrario seguiría disminuyendo el caudal del río. En la narrativa histórica de los comuneros, el río había dejado de ser aquel afluente capaz de abastecer a las fábricas textiles que se habían establecido en la cuenca desde finales del siglo XIX, para convertirse en un riachuelo moribundo a punto de secarse en temporada de estiaje. En las entrevistas sostenidas con los comuneros años después de su participación en los talleres de planeación, estos coincidieron en señalar la tendencia creciente de la degradación ambiental del bosque y del río, incluso con reforestaciones inadecuadas realizadas por las dependencias gubernamentales. La transformación más importante en su discurso consistió en significar la implementación de obras hidráulicas en el río como una estrategia de las autoridades para robarles el agua. Para los comuneros, el gasto del río ha disminuido no sólo por la falta de manejo forestal: ha sucedido también por el crecimiento urbano de la zona, que se ha llevado el agua a otra parte. Nuevamente subrayaron la transferencia de los servicios hídricos que genera su territorio hacia 
la ciudad, en este caso para los nuevos desarrollos inmobiliarios que se están construyendo en la zona.

- La depredación ambiental del turismo y el crecimiento urbano. Los comuneros atribuyeron una fuerte presión al bosque y al río por las actividades que realizan los turistas en el parque Los Dinamos y por el crecimiento urbano visible en los asentamientos humanos irregulares. Los turistas inciden en la contaminación de las partes altas porque el bosque no tiene una delimitación física que permita controlar el flujo de personas y sus respectivas actividades. Por otra parte, los comuneros señalaron a los asentamientos irregulares como los principales responsables del vertimiento de aguas residuales al cauce del río Magdalena, incluso en lugares ubicados antes de la planta potabilizadora. Algunos entrevistados aceptaron que el crecimiento de los asentamientos irregulares ha sido resultado de la venta ilegal de terrenos que realizan miembros de su comunidad, pero comentaron que esta práctica se ha realizado con la connivencia de las autoridades delegacionales. En ese sentido, identificaron como responsable directo de la degradación ambiental a los diferentes niveles de Gobierno (GDF y delegaciones) por su falta de compromiso real en la recuperación ambiental del río, así como por la omisión de su parte que permite la proliferación de procesos que alteran el equilibrio de la cuenca (asentamientos irregulares) y acciones perjudiciales al manejo forestal (las reforestaciones inadecuadas). Años después de haber concluido los espacios deliberativos, los comuneros seguían identificando al Gobierno (Sedema, delegación La Magdalena Contreras y Comisión de Recursos Naturales -Corena-), a los turistas y a los asentamientos irregulares como los principales responsables de la contaminación del río. No obstante, con el paso del tiempo, asumieron parte de la responsabilidad. En esta reflexión autocrítica, los comu- 
neros evaluaron que están poco unidos para defender el suelo de conservación, además de que cada vez son menos los miembros que se involucran en estas tareas. Se refirieron a una falta de sentido de pertenencia, y a un olvido de la lucha histórica que previamente había dado como resultado el reconocimiento jurídico del río y el bosque como elementos fundamentales de su territorio bajo la figura de bienes comunales. Además de este resquebrajamiento comunal, consideraron que el grupo que se dedica al comercio en el parque Los Dinamos privilegia su interés y rentabilidad económica sobre la conservación del bosque.

\subsection{El marco de los actores urbanos}

El marco de los actores urbanos sobre el río depende en gran medida de su proximidad física al cauce y del conocimiento que tienen en temas ambientales. Este último tiene un peso muy importante en la manera en que enmarcan la relevancia del proyecto. Por ejemplo, los participantes del taller dirigido a vecinos de las colonias de Chimalistac y Francisco Sosa no circunscribieron su análisis a los problemas que aquejan a la delegación en la que habitan (Coyoacán), sino que demostraron una preocupación y un conocimiento sobre la situación ambiental de toda la ciudad. Incluso, algunos participantes se refirieron a otras experiencias internacionales en la recuperación de ríos urbanos que podrían funcionar como modelo para el plan maestro. Los principales componentes de su marco cognitivo detectados son los siguientes:

- El río Magdalena como uno más de la cuenca. Los participantes señalaron la existencia de otros ríos en la ciudad (Mixcoac, San Buenaventura, Santo Desierto) que también se pueden recuperar. Esto no implicaba negar la importancia del río Magdalena, pero colocaba su recuperación como un proyecto que después tendría 
que replicarse en otros ríos. Desde el punto de vista de los comuneros, este elemento del marco cognitivo urbano mostraba que en los citadinos no había una apropiación afectiva del río y este tampoco constituía un elemento de identidad colectiva como sucedía en la población de Magdalena Atlitic. Ante la dificultad que tuvo el Gobierno para ejecutar algunos proyectos, ${ }^{7}$ varios participantes de los espacios deliberativos empezaron a mostrar un gran escepticismo sobre la factibilidad de recuperar el río Magdalena. A tal grado llegó esta valoración, que terminaron considerando más viable enfocarse en recuperar otro río de la ciudad sobre el que hubiera menos conflicto social. Lo importante era recuperar un río, aunque la primera experiencia exitosa no fuera la del Magdalena.

- El río como un problema sanitario. Los actores urbanos significaron el río principalmente como un problema ambiental y sanitario de la zona por su alta contaminación, lo que representaba un riesgo para la salud de los vecinos más próximos al cauce. Para muchos miembros de este sector, el río era prácticamente un drenaje a cielo abierto. El desarrollo urbano le había dado la espalda $y$, en vez de integrarlo como un elemento estructurador del paisaje urbano, lo había convertido en un depósito de desechos. Por esta razón, la principal demanda de los actores urbanos consistió en solucionar definitivamente este problema sanitario. Una vez saneado el cauce, podría promoverse la recuperación del río como un espacio público seguro y agradable, propicio para

7. Los comuneros se opusieron a la incorporación de colectores marginales en el suelo de conservación porque consideraron que el Sistema de Aguas de la Ciudad de México (SACM) no les había pedido permiso para realizar esta intervención en su territorio, y los vecinos de Chimalistac se opusieron a la construcción de una planta de tratamiento en la zona por el riesgo que representaba un manejo inadecuado de los lodos, lo que generaría efectos nocivos en su salud y en el patrimonio arquitectónico de la colonia. 
la recreación y la convivencia social. Algunos actores advirtieron que la creación de estos espacios públicos no sería una tarea sencilla porque la planicie de inundación estaba prácticamente invadida en su totalidad por viviendas, y calificaban como una verdadera utopía la recuperación del tramo entubado por la importancia que representa este tramo para conectar avenidas de alta circulación automovilística. Tiempo después de los talleres, los actores urbanos insistieron en que el río constituía un problema sanitario de la ciudad debido a su alto grado de su contaminación. Las fallas en la implementación del plan maestro y la falta de apertura a un mayor diálogo sobre aceptar el tratamiento de aguas por parte de varios vecinos aumentaron la interpretación de varios entrevistados de que el destino más adecuado para el río era su entubamiento para terminar con el hedor asociado a la contaminación del cauce.

- La falta de educación ambiental como responsable de la contaminación. Los actores urbanos que participaron en los espacios deliberativos para la recuperación del río Magdalena consideraron que los principales responsables de su contaminación eran las autoridades de Gobierno, los propios vecinos y los asentamientos irregulares. Las primeras, por su falta de capacidad para solucionar los problemas ambientales que aquejan a la ciudad o incluso por su falta de interés respecto a incorporar el tema ambiental como una de sus principales políticas de gobierno. Por su parte, a los vecinos y a los asentamientos irregulares les imputaron prácticas contrarias a la conservación del río, como arrojar basura y drenaje al cauce, además de una falta de interés general en recuperarlo. Hubo un énfasis importante en considerar que los tres actores adolecían de poca educación ambiental, aunado esto al sistemático incumplimiento de la ley. Una interpretación radical de este 
hecho consistía en relacionar la falta de una educación ambiental sostenible con un problema civilizatorio que impedía (e impedirá) tener un río limpio por la carencia de herramientas que le permitan a la ciudadanía tener un mayor conocimiento sobre los problemas ambientales -particularmente, hídricos- que aquejan a la ciudad, así como una competencia cívica para participar en la solución de los mismos de manera democrática.

\section{Conclusiones}

El caso del río Magdalena muestra cómo en la recuperación de ríos urbanos coexisten diferentes marcos cognitivos que no son fáciles de conciliar. Los actores tienen diagnósticos diferenciados sobre los problemas que aquejan a la cuenca, y tampoco coinciden plenamente en la atribución de responsabilidades detrás del deterioro ambiental. En principio, resulta complicado que algún actor rechace la idea de la recuperación: todos suelen coincidir en este principio regulativo (Perló y González, 2009), pero cuando este planteamiento se expresa en una iniciativa en particular, este acuerdo básico parece ser insuficiente para llevarla a cabo.

La disonancia cognitiva entre actores rurales y urbanos no es exclusiva de la cuenca del río Magdalena. Algunos estudios apuntan a que recurrentemente los actores rurales consideran que las ciudades son las principales beneficiadas de un río limpio porque este se suma a sus necesidades de abastecimiento, aunque esto se hace a expensas de disminuir o negar completamente la dotación que recibían para su propio consumo (Holt, Moug y Lerner, 2012; Mao, et al., 2015; Mostert, et al., 2007; Neaera Abers, 2007). En nuestro caso de estudio, la percepción de los comuneros de La Magdalena Atlitic sobre que los principales beneficios ambientales de su territorio (agua potable y aire limpio) son aprovechados por los actores urbanos se agrava por el 
entramado jurídico vigente que les impide cualquier tipo aprovechamiento forestal. Además, el propio bosque se encuentra bajo una permanente amenaza por el avance del crecimiento urbano.

Ante la diversidad de marcos, la viabilidad sociopolítica de estas iniciativas ambientales descansa en gran medida en poder construir una comunidad epistémica entre los diferentes actores para que puedan tejer acuerdos sobre la manera de concebir el problema y las estrategias para solucionarlo. En principio, estos acuerdos serán resultado de abrir espacios deliberativos en donde los diferentes interesados expresen su voz, completen su información sobre los procesos del territorio y conozcan las necesidades del resto de los participantes.

Como se pudo apreciar en el caso del río Magdalena, el funcionamiento de los espacios deliberativos no necesariamente desemboca en la creación de una comunidad epistémica; por el contrario, las disonancias entre los diferentes marcos cognitivos se pueden ampliar. Por ejemplo, tiempo después de los talleres de participación, los comuneros agregaron a su marco cognitivo la percepción de que las autoridades delegaciones se robaban el agua para dárselas a los desarrollos inmobiliarios de la zona, los actores urbanos aumentaron su escepticismo sobre la factibilidad de recuperar el río y las autoridades de Gobierno mostraron un cierto desencanto sobre la participación ciudadana como mecanismo de cambio para transformar la relación que la ciudad ha sostenido con sus ríos.

El caso fallido del río Magdalena reafirma que la construcción de comunidades epistémicas es el resultado de un proceso de largo aliento y no de reuniones esporádicas, casi limitadas a un cronograma de trabajo con tiempos establecidos bajo criterios estrictamente administrativos. En otras palabras, más que favorecer la emergencia de una comunidad epistémica, surgen obstáculos cuando las autoridades 
del Gobierno asumen que los espacios deliberativos tienen que ajustarse a un número predeterminado de reuniones, independientemente de los resultados obtenidos. Promover la participación bajo esta óptica soslaya que la construcción de acuerdos implica costos de transacción, máxime cuando la ciudadanía tiene una fuerte desconfianza institucional resultada de una serie de promesas no cumplidas en el pasado o de que los espacios deliberativos en iniciativas previas no han tenido una verdadera repercusión en la toma de decisiones.

En cambio, si los espacios deliberativos se mantienen a lo largo del tiempo, pueden dar paso a una comunidad epistémica que tenga una mayor certeza en las reglas de coordinación y distribución de responsabilidades entre los diferentes actores para resolver un problema (Kickert, Klijn y Koppenjan, 1997; Van Bueren, Klijn y Koppenjan, 2003).

Si un grupo de actores interesados en el rescate de un río se reúne durante poco tiempo, esta sola acción podría tener ciertos efectos en los marcos cognitivos de los participantes (ampliar la información disponible sobre la cuenca, o conocer una valoración distinta del problema), pero difícilmente desembocará en una red social comprometida con el mantenimiento de una política pública, ya que una vez cerrados los espacios deliberativos cada actor volvería a pensar el problema desde sus propias necesidades y a intentar resolverlo con sus propios recursos.

Por el contrario, si se crea una comunidad epistémica, entonces se sientan las condiciones para que los diferentes participantes tengan mayores incentivos para cooperar entre sí gracias a un mayor conocimiento de sus interlocutores -por ejemplo, la reputación obtenida por cada participante gracias al cumplimiento de sus compromisos-y a un creciente sentido de corresponsabilidad en la configuración y solución del problema (Schneider, et al., 2003). El mantenimiento de una comunidad epistémica parece clave para que la recuperación de ríos urbanos sea una iniciativa que 
logre trascender los periodos administrativos de Gobierno, ya que esta no descansaría únicamente en los intereses y propuestas de las autoridades en turno, sino que estaría apoyada en una red más amplia en donde diferentes actores presionarían a la siguiente administración para darle continuidad a la política.

En gran medida, el truncamiento en la integración de una comunidad epistémica en torno al río Magdalena permite comprender por qué su rescate, hoy en día, es una iniciativa olvidada no sólo por el Gobierno capitalino, sino también por una gran parte de los habitantes de la cuenca. $\varepsilon$

Bibliografía

Allison, G.,y Zelikow, P. (1999). Essence of Decision. Explaining the Cuban Missile Crisis. Nueva York: Longman.

Augé, M. (200I). Los "no lugares". Espacios del anonimato. Una antropología de la sobremodernidad. Barcelona: Gedisa.

Azuela,A., y Cosacov, N. (20I3).“Transformaciones urbanas y reivindicaciones ambientales. En torno a la productividad social del conflicto por la construcción de edificios en la ciudad de Buenos Aires”. EURE (Santiago), 39( I I8), |49-172.

Bateson, G. (20II). Espíritu y naturaleza. Buenos Aires: Amorrortu.

Bernhardt, E., y Palmer, M.A. (2007). “Restoring streams in an urbanizing world”. Freshwater Biology, 52(4), 738-75 I. doi: http://doi.org//0.1 I I I/j. I365-2427.2006.017/8.x

Bonnemaison, J. (198I). "Voyage autour du territoire". L’Espace Géographique, 4, I87-197.

Bunge, M. (1999). Buscar la filosofia en las ciencias sociales. México: Siglo XXI.

Burningham, K., y Cooper, G. (1999). "Being constructive: Social constructionism and the environment". Sociology, 33(2), 297-316. 
Clifford, N.J. (2007)." "River restoration: Paradigms, paradoxes Bibliografía and the urban dimension". Water Science \& Technology: Water Supply, 7(2), 57-68. doi: http://doi.org//0.2166/ ws.2007.04 I

Cortés, F. (2008). "Algunos aspectos de la controversia entre la investigación cualitativa e investigación cuantitativa", en F. Cortés, A. Escobar, y M. González de la Rocha (eds.), Método científico y política social. A propósito de las evaluaciones cualitativas de programas sociales (pp. 27-58). México: El Colegio de México.

Coser, L. (196I). Las funciones del conflicto social. México: Fondo de Cultura Económica.

Cruz, Y. (20 de julio de 2016). "Exigen liberar cauce". Reforma, p. 7.

Everard, M., y Moggridge, H. L. (20I2). "Rediscovering the value of urban rivers”. Urban Ecosystems, I 5(2), 293-3I4. doi: http://doi.org/ I0.1007/s | | 252-0 I I-0 I74-7

Findlay, S. J., y Taylor, M. P. (2006). "Why rehabilitate urban river systems?". Area, 38(3), 312-325. doi: http://doi. org/I0. I I I I/j. I 475-4762.2006.00696.x

Fung, A., y Wright, E. O. (200I). "Deepening Democracy: Innovations in Empowered Participatory Governance". Politics and Society, 29(I), 5-4I.

Funtowicz, S., y Ravetz, J. R. (1993). "Science for the post normal age". Futures, 25, 739-755.

García, R. (2000). El conocimiento en construcción. De las formulaciones de Jean Piaget a la teoría de los sistemas complejos. Barcelona: Gedisa.

Giannachi, G., y Stewart, N. (2005). Performing Nature. Explorations in Ecology and the Arts. Berna: Peter Lang.

Giddens, A. (20I0). La política del cambio climático. Madrid: Alianza.

Giménez, G. (200I).“Cultura, territorio y migraciones.Aproximaciones teóricas”. Alteridades, I I (22), 5-I4. 
Bibliografía
Goffman, E. (1975). Frame analysis. Los marcos de la experiencia. Madrid: Centro de Investigaciones Sociológicas, Siglo XXI.

Goldman, M., y Schurman, R.A. (2000). "Closing the "Great Divide': New Social Theory on Society and Nature". Annual Review of Sociology, 26, 563-584.

González, A., Hernández, L., Perló, M., y Zamora, I. (2010). "De las avenidas de agua a los ríos de asfalto", en A. González, L. Hernández, M. Perló, y I. Zamora (eds.), Rescate de ríos urbanos (PP. 16-34). México: PUEC, UNAM. González de León, T., Kalach, A., Lipkau, G., et al. (2010). México. Ciudad futura. México-Barcelona: RMVerlag.

Gough, C., y Shackley, S. (200I). "The Respectable Politics of Climate Change: The Epistemic Communities and NGOs". International Affairs, 77(2), 329-346. doi: https:// doi.org/I0. I I I I/I468-2346.00I95

Gurnell, A., Lee, M., y Souch, C. (2007). “Urban Rivers: Hydrology, Geomorphology, Ecology and Opportunities for Change". Geography Compass, I (5), I I I 8- I I 37. doi: http://doi.org/ I 0. I I I //j. I749-8I98.2007.00058.x

Haas, P.M. (1992).“Introduction: Epistemic communities and international policy coordination”, en P. M. Haas (ed.), Knowledge, Power, and International Policy Coordination (pp. I-37). Columbia, Carolina del Sur: University of South Carolina Press.

(2004). "Science policy for multilateral environmental governance”, en N. Kanie, y P.M. Haas (eds.), Emerging forces in environmental governance (Pp. I I5-I36). Tokio: United Nations University Press.

Hajer, M.,yWagenaar, H. (2003).“Introduction”, en M. Hajer y H.Wagenaar (eds.), Deliberative Policy Analysis. Understanding Governance in the Network Society (pp. I-30). Cambridge, Reino Unido: Cambridge University Press. Heim, W. (2003). "Slow activism: Homelands, love and the lightbulb", en B. Szerszynski, W. Heim, y C. Waterton 
(eds.), Nature performed. Environment, Culture and PerforBibliografía mance (pp. 183-202). Inglaterra: Blackwell.

Holt, A. R., Moug, P., y Lerner, D. N. (20I2). “The Network Governance of Urban River Corridors". Ecology and Society, I 7(4), 25. doi: http://doi.org/dx.doi.org//0.575 I/ ES-05200- 170425

Huitema, D., Mostert, E., Egas, W., et al. (2009). “Adaptative water governance: Assesing the institutional prescriptions of adaptative (co-)management from a governance perspective and defining a research agenda". Ecology and Society, I 4(I), 26.

Jaspers, F. G.W. (2003). “Institutional arrangements for integrated river basin management”. Water Policy, 5(I), 77-90. Kickert,W.J. M., Klijn, E. H.,y Koppenjan,J. F.M. (eds.) (1997). Managing complex policy networks. Londres: Sage.

King, G., Keohane, R. O., y Verba, S. ( 1994). Designing Social Inquiry: Scientific Inference in Qualitative Research. Nueva Jersey: Princeton University.

Kuhn, T. S. (I99I). "The Natural and the Human Sciences", en D. R. Hiley, J. Bohman, y R. Shusterman (eds.), The Interpretative Turn: Philosophy, Science, Culture (pp. 17-24). Ithaca: Cornell University Press.

Legorreta, J. (2009). Ríos, lagos y manantiales del Valle de México. Mexico: UAM, SMA-GDF.

Mao, X.,Yuan, D.,Wei, X., et al. (20I5).“NetworkAnalysis for a Better Water Use Configuration in the Baiyangdian Basin, China". Sustainability, 7, 1730- 174I. doi: http://doi. org/ I0.3390/su702I730

Martínez Alier, J. (2009). El ecologismo de los pobres. Conflictos ambientales y lenguajes de valores. Barcelona: Icaria.

McCully, P. (200I). Silenced rivers: The ecology and politics of large dams. Londres: Zed Books.

Melé, P. (2006). La producción del patrimonio urbano. México: CIESAS. 
Bibliografía
Mostert, N., Pahl-Wostl, C., Rees,Y., et al. (2007)."Social Learning in European River-Basin Management: Barriers and Fostering Mechanisms from Ten River Basins”. Ecology and Society, I 2(I), 19.

Neaera Abers, R. (2007). “Organizing for Governance: Building Collaboration in Brazilian River Basins”. World Development, 35(8), I450-I463. doi: http://doi.org//0.1016/j. worlddev.2007.04.008

Norwood, R. H. (1969). Patterns of discovery. Cambridge: Cambridge University Press.

ONU (2015). Aprobación del Acuerdo de París. Convención marco sobre el cambio climático. París: ONU. Recuperado de: https://goo.gl/sDBuVD

Perló, M., y González, A. (2009). “El papel de la interdisciplinariedad científica y de la participación social en el rescate de ríos urbanos", en I. Sandré, R. Luiz do Carmo, S. Vargas, y N. B. Guzmán (eds.), Gestión del agua: Una visión comparativa entre México y Brasil (pp. 177-188). Morelos: Archivo Histórico del Agua, Instituto Mexicano de Tecnología del Agua y Universidad Autónoma del Estado de Morelos.

Petts, G. E., Heathcote, J., y Martin, D. (eds.) (2002). Urban Rivers: Our Inheritance and Future. Londres: IWA Publishing. Postel, S., y Richter, B. (2003). Rivers for Life: Managing Water For People And Nature. Washington: Island Press.

Riley,A. L. (1998). Restoring Streams in Cities:A Guide for Planners, Policymakers, and Citizens.Washington: Island Press.

Rittel, H., y Webber, M. (1973). "Dilemmas in a General Theory of Planning”. Policy Sciences, 4, I55-169.

Sawyer, R. K. (2005). Social Emergence. Societies as Complex Systems. Nueva York: Cambridge University Press.

Schmoldt, D. L., Kangas, J., y Mendoza, G. (200I).“'Basic Principles of Decision Making in Natural Resources and the Environment", en D. L. Schmoldt, J. Kangas, G. Mendoza, y M. Pesonen (eds.), The Analityc Hierarchy Process in 
Natural Resource and Environmental Decision Making (pp. I - I3). Dordrecht: Kluwer Academic Publishers.

Schneider, M., Scholz, J., Lubell, M., et al. (2003). "Building Consensual Institutions: Networks and the National Estuary Program". American Journal of Political Science, 47(I), I42-I57.

Schön, D., y Rein, M. (1994). Frame Reflection: Toward the Resolution of Intractable Policy Controversies. Nueva York: Basic Books.

Searle, J. (1997). La construcción de la realidad social. Barcelona: Paidós.

Sedema y UNAM (2009). Plan de manejo integral y aprovechamiento sustentable de la cuenca del río Magdalena. México: Secretaría del Medio Ambiente del Gobierno del Distrito Federal.

Strauss, A., y Corbin, J. (2002). Bases de la investigación cualitativa. Técnicas y procedimientos para desarrollar la teoría fundamentada. Medellín: Universidad de Antioquia.

Thoms, M. C., Gilvear, D. J., Greenwood, M.T., y Wood, P. J. (2016)."An introduction to river science: research and applications", en D. J. Gilvear, M. T. Greenwood, M. C. Thoms, y P. J. Wood (eds.), River Science. Research and Management for the 2 Ist Century (Pp. I-14). Chichester: John Wiley \& Sons Inc.

Tucci, C. E. M. (20I2). Gestão da drenagem urbana. BrasiliaMéxico: CEPAL.

Van Bueren, E. M., Klijn, E.H., y Koppenjan, J. F. M. (2003). "Dealing with Wicked Problems in Networks: Analyzing and Environmental Debate from Network Perspective". Journal of Public Administration Research and Theory, I3(2), 193-212.

Wagenaar, H. (2006). “Democracy and Prostitution”. Administration \& Society, 38(2), 198-235.

Wallen, R. (2012). “Ecological Art: A Call forVisionary Intervention in a Time of Crisis". Leonardo, 45(3), 234-242. 
Bibliografía

Warner, J., Wester, P., y Bolding, A. (2008). "Going with the flow: River basins as the natural units for water management?". Water Policy, 10(S2), I2I-138. doi: 10.2166/ wp.2008.210

Warren, M. E. (1992). "Democratic Theory and Self-Transformation". American Political Science Review, 86 (I), 8-23. doi: http://doi.org/http://dx.doi.org//0.2307//9640I2

White, R. (1995). The Organic Machine. The Remarking of the Columbia River. Nueva York: Hill \& Wang.

Woodgate, G., y Redclift, M. (1998). "From a sociology of nature to environmental sociology: beyond social construction". Environmental Values, 7(I), 3-24.

Worster, D. (1992). Rivers of Empire. Water, Aridity, and the Growth of the American West. Nueva York: Pantheon.

Zamora, I. (20I6). "Arte, representaciones sociales y recuperación de ríos urbanos", en E. S. Martins Freitas, y J.E. Castro (eds.), Water discourses and representations:Their significance and impact in public policies and social practices. Working Paper Series (pp. 34-59). Newcastle Upon Tyne-Buenos Aires-Belo Horizonte:Waterlat-GOBACIT Research Network. doi: I0.I 3 |40/RG.2.2.2I 784.39688 